\title{
Altered Levels of Gq Activity Modulate Axonal Pathfinding in Drosophila
}

\author{
Anuradha Ratnaparkhi, Santanu Banerjee, and Gaiti Hasan \\ National Center for Biological Sciences, Tata Institute of Fundamental Research, Bangalore 560065, India
}

A majority of neurons that form the ventral nerve cord send out long axons that cross the midline through anterior or posterior commissures. A smaller fraction extend longitudinally and never cross the midline. The decision to cross the midline is governed by a balance of attractive and repulsive signals. We have explored the role of a G-protein, $\mathrm{G} \alpha \mathrm{q}$, in altering this balance in Drosophila. A splice variant of $\mathrm{G} \alpha \mathrm{q}, \mathrm{dgq} \alpha 3$, is expressed in early axonal growth cones, which go to form the commissures in the Drosophila embryonic CNS. Misexpression of a gain-of-function transgene of $d g q \alpha 3$ (AcGq3) leads to ectopic midline crossing. Analysis of the AcGq3 phenotype in roundabout and frazzled mutants shows that AcGq3 function is antagonistic to Robo signaling and requires Frazzled to promote ectopic midline crossing. Our results show for the first time that a heterotrimeric G-protein can affect the balance of attractive versus repulsive cues in the growth cone and that it can function as a component of signaling pathways that regulate axonal pathfinding.

Key words: dgq; Robo; Frazzled; Netrins; G-protein; midline; axon guidance
Axons use cues present at different choice points in the cellular environment to reach their targets. These cues are attractive and repulsive in nature and function at short range by contact or through long range by diff usion (Tessier-Lavigne and Goodman, 1996). The midline of the CNS in vertebrates and invertebrates functions as one such choice point for axons that need to project to their targets on the opposite side in the CNS. Cells at the midline provide cues that are both attractive and repulsive and thus enable axons to make appropriate decisions at the midline. Attractive cues are encoded by molecules called Netrins (Ishii et al., 1992; Kennedy et al., 1994; Harris et al., 1996; Mitchell et al., 1996), whereas repulsive cues are encoded by a class of molecules called Slit. These cues and their receptors Frazzled/DCC and the Roundabout (Robo) family, respectively, are also highly conserved (Chan et al., 1996; Keino-Masu et al., 1996; Kolodziej et al., 1996; Kidd et al., 1998a; Rajagopalan et al., 2000a,b; Simpson et al., 2000a,b). Studies in Drosophila and vertebrates have shown that attraction and repulsion are a consequence of the signaling pathways triggered by the two receptors and are not intrinsic to the nature of the individual ligands. (Bashaw and Goodman, 1999; Hong et al., 1999). Studies in Drosophila have also shown that all cells in the nervous system are competent to respond to attractive and repulsive cues, thereby suggesting that the balance between attraction and repulsion determines the final response of the growth cone (Bashaw and Goodman, 1999). This is achieved in part by regulating expression of individual receptors (Kidd et al., 1998a) and in part by modulating the activity of the signaling pathways (Menon and Zinn, 1998; Bonkowsky et al., 1999; Sun et al., 2000; Bashaw et al., 2000). Thus, identifying the signaling

\footnotetext{
Received Nov. 8, 2001; revised Feb. 4, 2002; accepted Feb. 20, 2002.
}

This work was supported by a grant from the Department of Biotechnology, Government of India, and core funding from National Center for Biological Sciences. We thank Mattias Landgraf for useful discussions and Veronica Rodrigues, Satyajit Mayor, and K. VijayRaghavan for critical comments on this manuscript.

Correspondence should be addressed to Gaiti Hasan, National Center for Biological Sciences, Tata Institute of Fundamental Research, Gandhi Krishi Vigyan Kendra Campus, Bellary Road, Bangalore 560065. E-mail: gaiti@ncbs.res.in. Copyright (C) 2002 Society for Neuroscience $0270-6474 / 02 / 224499-10 \$ 15.00 / 0$ pathways and their regulation in response to various cues is important in understanding how this balance is achieved and maintained.

In vitro studies in vertebrate systems have shown that altering cyclic nucleotide levels and calcium in the growth cone can convert attraction into repulsion (Song et al., 1997; Hong et al., 2000; Zheng, 2000), suggesting that G-protein-coupled signaling pathways are involved in this process. In this study we have examined the role of the heterotrimeric G-protein Gq in growth cone guidance in Drosophila. The gene $d g q$ encodes the $\alpha$ subunit of the Gq class of heterotrimeric G-proteins in Drosophila. This family of G-proteins is known to activate the phosphoinositide cascade within cells, which involves generation of inositol $1,4,5$,trisphosphate $\left(\mathrm{IP}_{3}\right)$ followed by release of intracellular calcium through the $\mathrm{IP}_{3}$ receptor (Exton, 1994). In Drosophila, the role of this gene in mediating phototransduction in the adult eye has been well established (Lee et al., 1994; Scott et al., 1995). We find that a splice variant of the Gq gene (dgq 23 ; Talluri et al., 1995; Alvarez et al., 1996) is expressed in the embryonic CNS during development. In this study we show for the first time that a dominant active form of $d g q \alpha 3$ modulates repulsive signaling in the growth cone, possibly in response to attractive cues. Our results suggest that Gq signaling could function as a part of the regulatory network that functions to tilt the balance from repulsion to attraction during midline crossing of axons.

\section{MATERIALS AND METHODS}

cDNA isolation and sequencing. Embryonic and appendage cDNA libraries were screened using a probe generated by a PCR using degenerate primers on an appendage library (Wang et al. 1999). The primer sequences were as follows: (1) 5'AC(T/C/A/G)TT(T/C)AT(T/C/A)AA(G/A)CA(A/G)ATG 3'; (2) 5'(A/G)AA(A/G)CA(A/G/T/C)TG(A/G/T)ATCCA(C/T)TT 3'.

They correspond to the conserved amino acid sequences "TFIKQM" and "KWIQHCF" in the helical domain of G $\alpha$ q proteins. Standard PCR conditions for degenerate primers were used (Hasan and Rosbash. 1992). The PCR product was reamplified using an internal primer: $5^{\prime} \mathrm{T}(\mathrm{T} / \mathrm{C})(\mathrm{A} /$ G)TC(A/G)AA(A/T/C/G)GG(A/G)TA(T/C)TC3', which corresponds to the conserved amino acid sequence "EYPFDL". A 400 bp product was obtained using the internal primer. This was subcloned into the plasmid 
pBluescript (Stratagene, La Jolla, CA) and sequenced. The sequenced clone was then used to screen appendage and embryonic cDNA libraries. cDNA clones obtained from the two libraries were sequenced manually (Sanger et al., 1977).

RT-PCR analysis. Poly $\left(\mathrm{A}^{+}\right)$RNA was isolated from various tissues following standard procedures (Sambrook et al., 1989). Primers complementary to exon 9 and 10 (see Fig. $1 A$ ) were used for RT-PCR analysis of $d g q \alpha 3$. Their sequences are as follows: PE-3 (exon 9): 5'AACTCGAGTACGATGGTCCTCAGCGAG 3' and PE-4 (exon 10): 5' AAGGATCCTCCAAATCCAGTTTAGACC 3'. Reverse transcription and PCR (RT-PCR) was according to published procedures (Sinha and Hasan, 1999).

In situ hybridization to whole mount embryos. In situ hybridization to embryos was according to the procedure described by Tautz and Pfiefle (1989). A 210 bp $d g q \alpha 3$-specific probe was generated and labeled by PCR using primers to exons 11 and 14. Digoxygenin-dUTP from Boehringer Mannheim (Mannheim, Germany) was included in the PCR mix.

Western blot analysis. Protein extracts from adult tissues and $0-8$, $8-16$, and 16-24 hr embryos were made by homogenization in polyacrylamide gel sample buffer at twice its normal concentration (Sambrook et al., 1989). The samples were run on a $10 \%$ SDS-polyacrylamide gel and transferred to nitrocellulose membranes. Detection of the protein blot was according to published procedures (Edery et al., 1994) with minor modifications. Anti-Gq antiserum from Santa Cruz Biotechnology (Santa Cruz, CA) was used at a dilution of 1:1000.

Immunohistochemical methods. Immunohistochemical staining of whole-mount embryos was according to published protocols (Gould et al., 1990). Developmental stages were identified following the description by Wieschaus and Nusslein-Volhard (1986). The anti-Gq antibody used from Santa Cruz Biotechnology has been raised against the C-terminal peptide (FAAVKDTILQLNLKEYNLV) of mammalian G $\alpha$ q. This differs from the corresponding Drosophila Dgq $\alpha 3$ sequence by a single residue (FAAVKDTILQSNLKEYNLV). For immunohistochemistry the antibody was used at a dilution of 1:200. Anti- $\beta$ gal monoclonal supernatant, monoclonal antibody (mAb) 40-1a (Developmental Studies Hybridoma Bank, University of Iowa, Iowa City, IA) and mAb 1D4 (anti-Fasciclin II; courtesy C. Goodman laboratory, University of California, Berkeley, CA) were used at a dilution of 1:25 each. Anti-Robo (courtesy C. Goodman) and anti-Connectin antiserum (courtesy of Rob White, Department of Anatomy, University of Cambridge, Cambridge, UK) were used at a dilution of $1: 10$. Vectastain $\mathrm{A}+\mathrm{B}$ kit (Vector Laboratories, Burlingame, CA) was used for nonfluorescent immunohistochemical visualizations. The stained embryos were filleted and mounted in $90 \%$ glycerol. Fluoro-isothiocyanate (FITC) and rhodamine or Alexa Red-conjugated secondary antibodies (Jackson ImmunoResearch, West Grove, PA; Molecular Probes, Eugene, OR) were used at a dilution of 1:200. Specimens stained with fluorescent secondaries were mounted in $70 \%$ glycerol containing $1 \mathrm{mg} / \mathrm{ml}$ of $p$-phenylenediamine (Sigma, St. Louis, MO) to prevent quenching.

Confocal imaging. Confocal images of antibody staining done with fluorescent secondaries were viewed on Bio-Rad (Poole, UK) MRC 1024. For double-labeled images, data from the two channels (605 DF32 for rhodamine and Alexa Red and 522 DF32 for FITC) were superimposed using Metamorph software version 4.0. Confocal sections of $2 \mu \mathrm{M}$ thickness were obtained, and composite images were created by merging relevant numbers of sections. Confocal sections of $0.3 \mu \mathrm{M}$ thickness were obtained for the images shown in Figure 2, $E$ and $F$.

Site-directed mutagenesis and germline transformation. The Q203L mutation requiring an $\mathrm{A} \rightarrow \mathrm{T}$ change was introduced in the $d g q \alpha 3 \mathrm{cDNA}$ by site-directed mutagenesis using the Quik-change kit by Stratagene. The primer used to introduce the mutation was 5'CGGTGGTCTGCGATCCG 3'. The mutant cDNA was sequenced fully to ensure that no other mutations had been incorporated into the modified sequence. Both mutant and wild-type cDNAs were independently subcloned into the transformation vector pUAST (Brand and Perrimon, 1993) to obtain germline transformants. Two independent transformant lines for each construct were obtained. These are $U A S-G q 3^{F F 17-2}$ on chromosome 3 , $U A S-G q 3^{M M 17-2}$ on chromosome $2, U A S-A c G q 3^{F 58 a}$ on chromosome 1, and $U A S-A c G q 3^{F 58 c}$ on chromosome 2. Equivalent phenotypes were observed with both sets of transformant lines.

Flystocks. All stocks were grown at $25^{\circ} \mathrm{C}$. The following $G A L 4$ stocks were used: C155-GAL4 (Lin and Goodman, 1994), ftz $z_{n g}-G A L 4$ and eve $_{n g}$-GAL4 (courtesy of Jim Jaynes, Thomas Jefferson University, Philadelphia, PA) (Baines et al., 1999). The ApC-taußgal stock was obtained from the laboratory of Dr. John Thomas (Salk Institute, San Diego, CA)
(Lundgren et al., 1995), whereas the $A p-G A L 4$ stock was obtained from the Drosophila stock center (Bloomington, IN). UAS-roboY-F (Bashaw et al., 2000) was obtained from C. Goodman's laboratory. The $D f(2 R) v g-C$ stock, which carries the deficiency for $d g q$, was obtained from the Drosophila stock center and placed against a CyoActßgal balancer to identify homozygous deficiency embryos. For expression of AcGq3, males of the genotype $U A S$-AcG $3 / F M 7-G F P$ were crossed to homozygous females of one of the following genotypes: (1) C155-GAL4, (2) C155-GAL4; +; Ap-taußgal, (3) +; +; ftz ng $^{-G A L 4, U A S-t a u \beta g a l, ~ o r ~(4) ~ U A S-t a u ß g a l ; ~}$ eve ${ }_{n g}-G A L 4$. To study the behavior of Apterous neurons, an ApterousGAL4/CyoWgßgal; Apterous-taußgal strain was generated and subsequently crossed to $U A S-A c G q 3^{F 58 c} / C y o A c t \beta g a l$. To examine the genetic interactions between $A c G q 3$ and robo ${ }^{1}$ and frazzled mutants, the following strains were generated: C155-GAL4; $\mathrm{ra}^{4} /$ CyoActßgal;+/+., UASAcGq3/FM7ftzßgal; fra ${ }^{3} /$ CyoActßgal; +/+., and UAS-AcGq3/FM7ftzßgal; robo $^{1} /$ CyoAct $\beta$ gal. Homozygous and heterozygous mutant embryos were distinguished based on the presence or absence of marked balancers in each case. For studying the interaction between roboY-F and $A c G q 3$, $U A S-A c G q 3^{F 58 c} / C y o-G F P, U A S-r o b o Y-F$ strain was generated and crossed to $C 155-G A L 4$ females. Expression of AcGq3 was confirmed by immunohistochemical staining with anti-Gq antibody. With both $C 155$ $G A L 4$ and $f t z_{n g}-G A L 4$ drivers, the pattern of Gq expression observed is different from the wild-type pattern.

\section{RESULTS}

\section{Identification and expression of dgq 33 in Drosophila embryos}

cDNA clones corresponding to the $d g q$ gene were isolated in library screens using a fragment from the eye-specific splice variant dgqa1 (Lee et al., 1990). We screened libraries derived from either embryo or appendage RNAs and analyzed $d g q$ positive cDNA clones by restriction digests and PCR. The three classes of cDNA clones obtained are shown in Figure $1 A$. Of these, one class corresponds, in the region of the open-reading frame, to the previously identified splice variant transcript of the $d g q$ gene, called $d g q \alpha 3$, known to be expressed in several adult tissues (Talluri et al., 1995; Alvarez et al., 1996). This class was isolated repeatedly from the embryo cDNA library, as judged by extensive PCR analysis. Specifically, primers to exons 4 and 9, 6 and 9, 6 and 11, and 11 and 14 (Fig. $1 A$ ), amplified the expected fragments of $673,321,445,210 \mathrm{bp}$. No amplification was observed using primers to exons 6 and 12 and exons 12 and 13, indicating that none of the embryonic cDNAs belong to the next class of cDNA isolated from the appendage library ( $d g q \alpha 4)$ (Fig. 1A). Because this was the first characterization of a $d g q$ transcript in Drosophila embryos, we performed RT-PCRs using dgqa3specific primers from exons 11 and 14 (Fig. 1A). As shown in Figure $1 B, d g q \alpha 3$-specific transcripts are present in $\operatorname{poly}\left(\mathrm{A}^{+}\right)$ RNA extracted from heads, appendages, male and female bodies, and embryos. These results were corroborated by a Northern blot analysis using the unique $3^{\prime}$ region of $d g q \alpha 3$ as a probe (data not shown). A third class of cDNA clones was found only in the appendage library and appeared identical to the adult visual $\mathrm{G} \alpha \mathrm{q}$

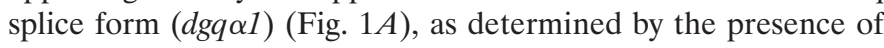
dgq $\alpha 1$-specific exon 7 (by PCR), and exons 10 and 13 (by sequencing) (Fig. 1A). We have not analyzed this cDNA any further.

Next we ascertained the presence of the Dgq $\alpha 3$ protein in Drosophila embryos by Western blot analysis of embryo extracts (Fig. $1 C$ ). The antiserum used recognizes the $\mathrm{C}$-terminal end of the mammalian Gq protein. In Drosophila $\mathrm{Gq}$ this $\mathrm{C}$-terminal sequence is conserved only in the Dgq $\alpha 3$ form (Fig. 1A, asterisk) (see Materials and Methods). The results obtained indicate that a $39 \mathrm{kDa}$ band, corresponding to the predicted size of the Dgq $\alpha 3$ protein, is present in embryos throughout development from as early as $0-8 \mathrm{hr}$. 
A
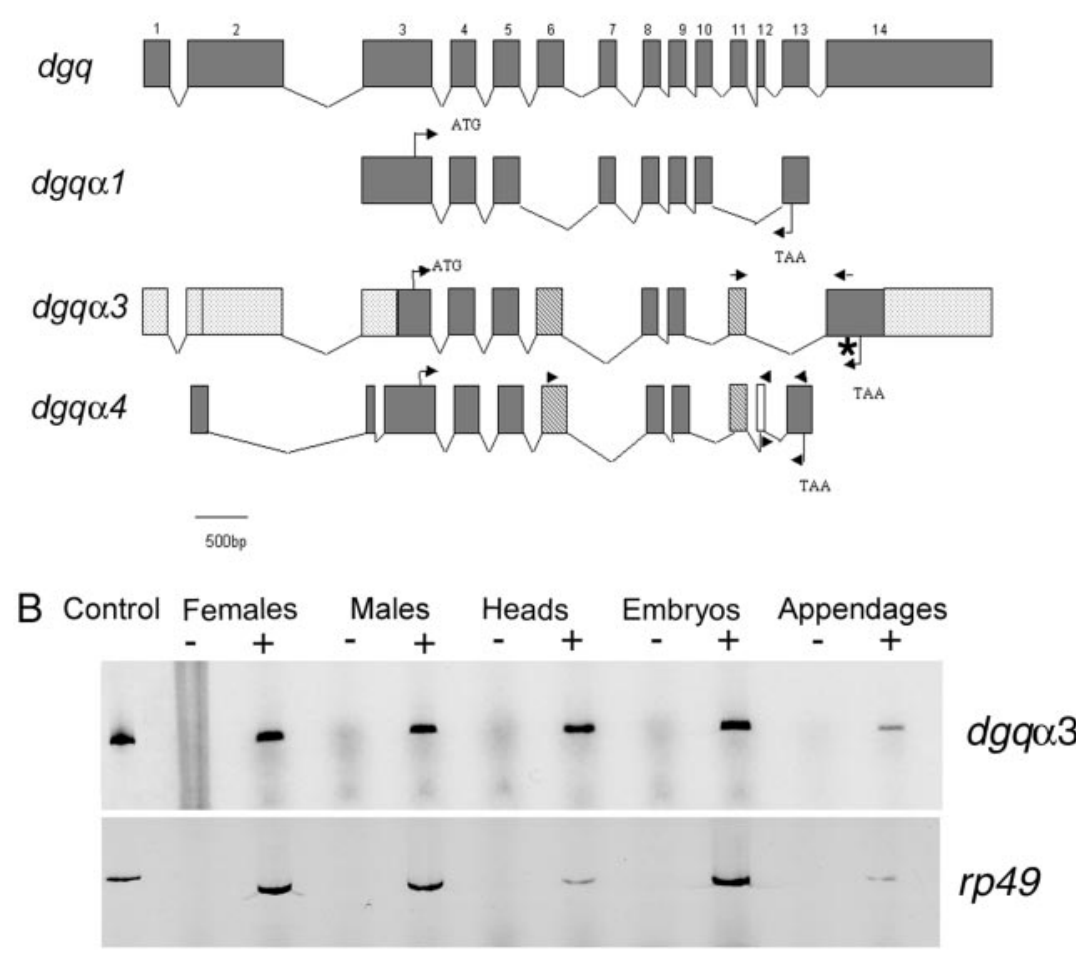

Figure 1. A, Structure of the $d g q$ gene and its splice variants $d g q \alpha 3$ and $d g q \alpha 4$. All known exons are numbered and shown as boxes in the row marked dgq. The hatched exons indicate regions unique to $d g q \alpha 3$ and $d g q \alpha 4$ splice variants. In the row marked $d g q \alpha 3$, the stippled exons indicate regions that are not found in the $d g q \alpha 3$ cDNAs identified by us. The open box for exon 12 indicates a $d g q \alpha 4$-specific exon. The arrows on $d g q \alpha 3$ indicate positions of the primers used for RT-PCR analysis shown below. The asterisk marks the $\mathrm{C}$-terminal region recognized by the Gq3 antiserum. Arrowheads on dgq $\alpha 4$ indicate primer positions used to differentiate it from $d g q \alpha 3$. $B$, Expression of $d g q \alpha 3$ mRNA by RT-PCR. A $d g q \alpha 3-$ specific band of $210 \mathrm{bp}$ is seen in poly $\left(\mathrm{A}^{+}\right)$RNA extracted from adult male and female bodies, heads, and appendages, as well as embryos. Lanes marked as (-) were loaded with PCR reactions from minus reverse transcriptase control tubes. The control lane contains a PCRamplified product from the $d g q \alpha 3$ cDNA clone. The quality and quantity of poly $\left(\mathrm{A}^{+}\right)$RNA isolated from each tissue was estimated by an RT-PCR done with primers specific to the rp49 gene. The rp49 control plasmid is a genomic clone, leading to small difference in its size from the RT-PCR product. $C$, Expression of $\operatorname{Dgq} \alpha 3$ protein. A Western blot of protein lysates made from staged embryos and adult heads was stained with antiserum to Gq. A 39 $\mathrm{kDa}$ band corresponding to the size of $\operatorname{Dgq} \alpha 3$ can be seen in all the lanes.

\section{dgq $\alpha 3$ RNA and protein are expressed predominantly in axonal tracts of the embryonic CNS}

Presence of $d g q \alpha 3$ RNA and protein in embryos suggests an involvement of the $d g q$ gene in Drosophila development. We therefore studied the expression pattern of $d g q \alpha 3$ during embryonic development by in situ hybridization with a $d g q \alpha 3$ splice variant-specific probe. Although $d g q \alpha 3$ RNA is present in earlier stages, tissue-specific expression of $d g q \alpha 3$ is first seen in the brain and ventral nerve cord at stage 13 (Fig. 2A). This expression persists till late in development, where in addition, strong expression is seen in an anterior sense organ (Fig. 2B). This organ corresponds in position to the Bolwig's organ or the larval eye (Schmucker et al., 1992). In similar experiments with a dgq 4 specific probe, no hybridization was observed to any region of the developing embryo (data not shown).

Expression of Dgq $\alpha 3$ during development of the embryonic nervous system was further confirmed by immunohistochemical staining of wild-type embryos with the Gq antiserum. The CNS in Drosophila embryos develops from a delaminated set of neuroblasts that derive from the ventral neuroepithelium after gastrulation (Goodman and Doe, 1993). These neuroblasts undergo a series of highly stereotyped cell divisions during embryonic stages 8-11, which lead to a well defined spatial pattern (Goodman and Doe, 1993). The expression of $\operatorname{Dgq} \alpha 3$ at these and earlier stages appeared diffuse and non-neuronal (data not shown). The first indication of Dgq $\alpha 3$ expression in the CNS is at early stage 12 (Fig. $2 C$ ). This is also the stage at which the pioneer neurons begin formation of axon pathways that give rise to the typical ladder-like appearance of the embryonic CNS, consisting of longitudinal tracts and anterior and posterior commissures that can be visualized with the axonal marker mAb BP102 (Fig. 2I). A similar pattern of expression of anti-Gq and the axonal marker mAb BP102 at early stage 12 suggests that $\operatorname{Dgq} \alpha 3$ is expressed in the pioneer growth cones that give rise to the commissures (Fig. $2 E, F)$ (Klambt et al., 1991). At later stages of development Dgq $\alpha 3$ protein expression increases in the axonal tracts of the CNS (Fig. 2D, $G$ ). In addition, Dgq $\alpha 3$ expression was visible in the midgut epithelium at stages 12 (Fig. 2C) and 13 (data not shown). Specificity of the anti-Gq antibody was determined by immunohistochemical staining of embryos that were either deficient for $d g q$ in one copy (Fig. 2G,I) or both copies (Fig. 2H,J). The likely presence of the Dgq $\alpha 3$ protein in growth cones of early commissural axons lead us to examine the role for this gene in axonal growth and guidance.

\section{Neuronal expression of the activated form of Dgq $\alpha 3$ causes abnormal midline crossing}

Axonal guidance in the Drosophila CNS requires the interpretation of both attractive and repulsive cues, generated by cells that lie in the midline (Harris et al., 1996; Kolodziej et al., 1996; Mitchell et al., 1996; Culotti and Merz, 1998; Kidd et al., 1999). The expression pattern of $\operatorname{Dgq} \alpha 3$ protein suggested that it might 

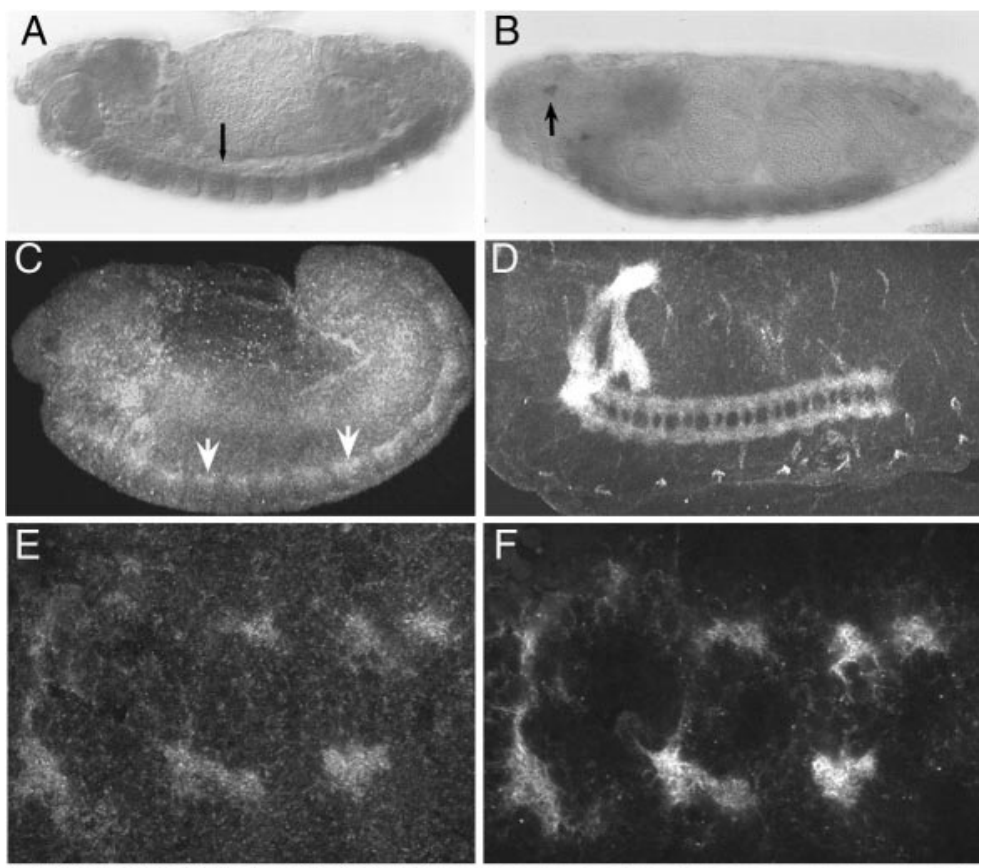

Figure 2. In situ localization of $d g q \alpha 3$ RNA and protein in Drosophila embryos. $A, B$, In situ hybridization of a dgqa3-specific probe showing RNA expression at late stage $13(A)$ and stage 16 $(B)$. The arrows indicate localization of $d g q \alpha 3$ RNA in the ventral nerve cord $(A)$ and the Bolwig's organ $(B)$. C, D, Dgq $\alpha 3$ expression in the embryonic CNS observed by immunofluorescent staining using anti-Gq antibodies at stage $12(C$, arrows) and stage $17(D)$. $E, F$, Confocal images of the developing CNS in an early stage 12 embryo after double-staining with anti-Gq $(E)$ and the axonal marker mAb BP102 $(F) . G, H$, Confocal images of the CNS from stage 15 embryos stained with Anti-Gq. Embryos were either heterozygous $(G)$ or homozygous $(H)$ for the $D f(2 R) v g$ - $C . I, J$, Embryos shown in $G$ and $H$ were double-stained with mAb BP102. Heterozygous and homozygous deficiency embryos were distinguished by the presence of actin-lacZ on the balancer chromosome, which shows up as green spots (I, arrow). The commissures are poorly formed and appear thin in $J$. Anti-Gq was visualized using rhodamine-labeled secondary antibodies, whereas an FITC-linked secondary was used for BP102. Magnification: $A-D, 200 \times ; E-J$, $600 \times . A-C$ are lateral views with dorsal side up. $D-J$ are ventral views. In all cases anterior is to the left.
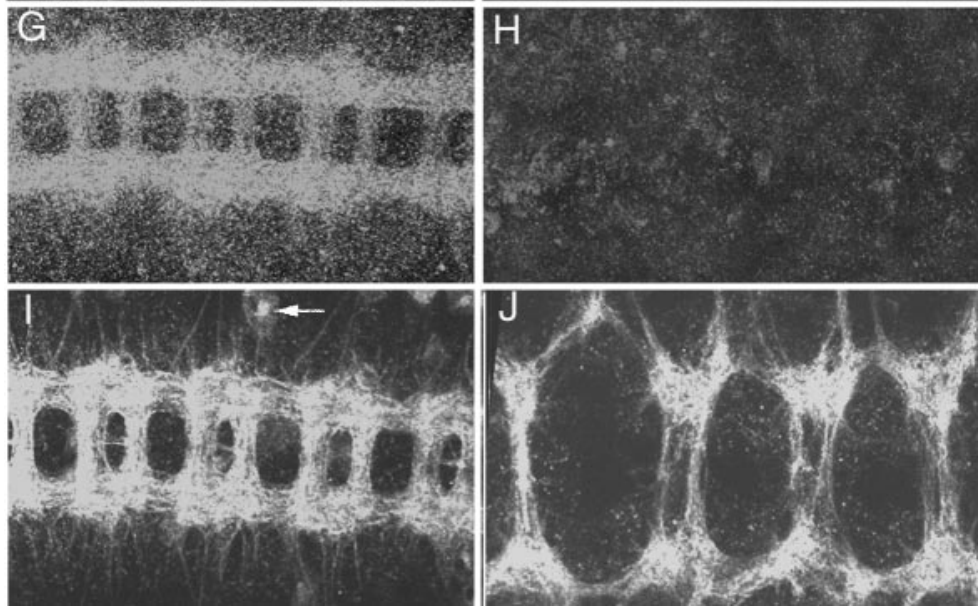

be required in early growth cones for the interpretation of these cues. To address this possibility, it was essential to alter $\mathrm{G} \alpha \mathrm{q}$ signaling in a tissue and cell-specific manner. We therefore created transgenic strains with a dominant active form of $\operatorname{Dgq} \alpha 3$, in which a glutamine residue at position 203 was mutated to a leucine. The mutation was made based on previous studies on dominant active forms of $\mathrm{G} \alpha \mathrm{q}$ from mammalian cells and Drosophila (DeVivo et al., 1992; Lee et al., 1994). As controls we also generated transgenic lines carrying the wild-type form of $\operatorname{Dgq} \alpha 3$.

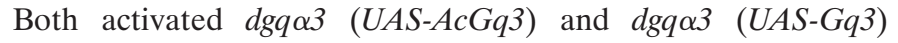
cDNAs were placed under the control of the GAL4-inducible $U A S$ promoter that would allow tissue and cell-specific expression. To study the effect of $U A S-A c G q 3$ expression on axonal development, we used the C155-GAL4 line initially, which expresses in all postmitotic neurons (Lin and Goodman, 1994). When stained with mAb BP102, the CNS of C155-GAL4; UAS$G q 3$ embryos looked normal (Fig. $3 A$ ). In embryos expressing AcGq3 3 the pattern of the CNS appeared mildly deranged in that the commissures were thicker, and the neuropil region was broader than usual (Fig. 3B). More significant differences between the two genotypes were obvious when a monoclonal antibody against Fasciclin II (mAb 1D4) was used (Fig. 3C-H). At stage 13, anti-Fasciclin II (anti-Fas II) marks the pioneer axons that go to form the first longitudinal axon pathway (Fig. 3C), which by stage 16 , defasciculates to form three distinct fascicles (Fig. $3 G$ ). These axons project ipsilaterally and do not cross the midline. In embryos of the genotype C155-GAL4; UAS-Gq3 this projection pattern was identical to wild-type embryos, indicating that overexpression of Dgq $\alpha 3$ has no effect on Fas II-expressing axons (Fig. 3C,E, $G$ ). However, in embryos expressing AcGq3, Fas II-positive axons appeared abnormal in all the embryos examined (Fig. 3D, $F, H$ ) with variations in the extent of abnormality. One obvious phenotype observed was that of "stalling" of Fas IIpositive axons, which could be seen clearly at late stage 13 (Fig. $3 D$, arrowheads). At this stage, minute outgrowths from the cell bodies and axonal tracts were also visible (Fig. $3 F$, arrowheads). Stage 15 onward, Fasciclin II-expressing axons could be seen crossing the midline (Fig. $3 H$, arrow). Occasionally a whirling phenotype similar to that observed in robo mutant alleles was seen (Fig. 3H, asterisk) (Kidd et al., 1998a). A quantification of these phenotypes is given in Table 1.

From these experiments the fate of the axons that cross the midline was unclear. For this purpose we generated a strain with the Apterous tau-Bgalactosidase (Ap-taußgal) construct in which 

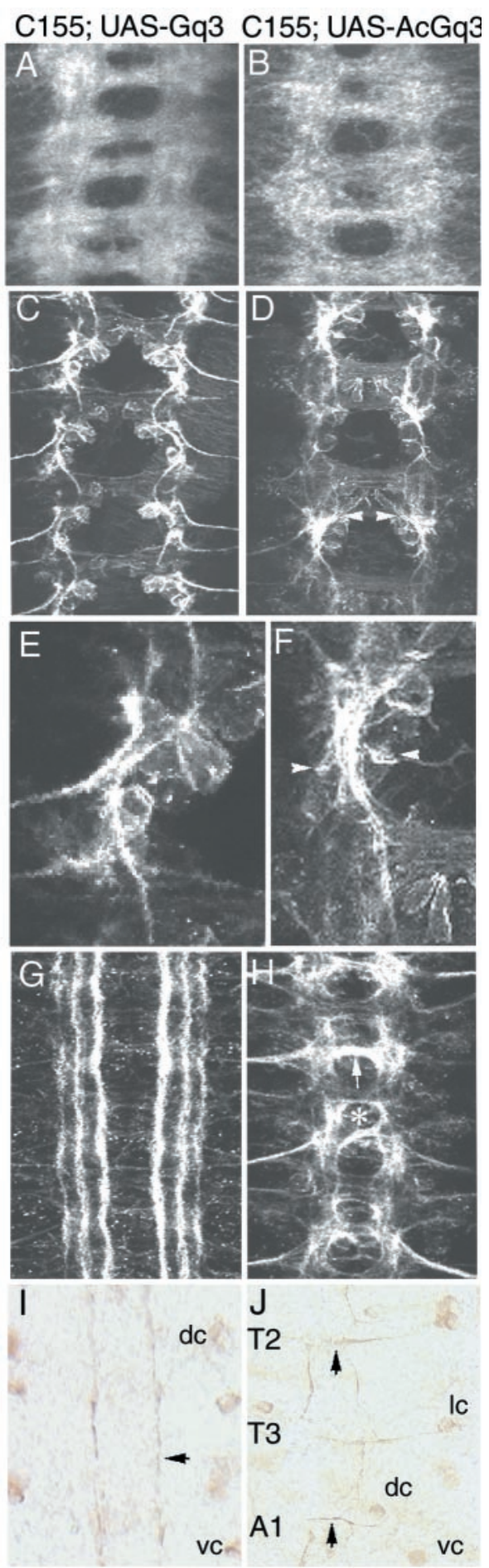

Figure 3. Expression of activated $\operatorname{Dgq} \alpha 3$ (AcGq3) in the CNS leads to defects in axonal growth and guidance. Embryos of the genotype C155GAL4; UAS-Gq3/+ or C155-GAL4/UAS-AcGq3 were stained with mAb BP102 ( $A, B$, stage 15) or mAb 1D4, which is specific for Fas II $(C-H)$. $C-F$, Late stage 13 . Fas II-expressing axons are abnormal in embryos expressing $\operatorname{AcGq} 3(D, F)$ as compared with the control embryos in $C$ and $E$. The arrowheads in $D$ indicate stalled axons, and the arrowheads in $F$ point toward minute outgrowths from neuronal cell bodies and axonal tracts. $E$ and $F$ are enlarged images from $C$ and $D$, respectively. At stage 15, Fas II-positive axons appear normal in $G$, whereas in $H$ they are seen crossing the midline (arrow) through the posterior commissure and recrossing through the anterior commissure (*). I, Anti- $\beta$ gal staining of a stage 16 embryo of the genotype C155-GAL4/+; UAS-Gq3/Ap-taußgal. Normal ipsilateral projection of Apterous-expressing axons is observed single axons could be observed. Ap-taußgal marks specific Apterous-expressing neurons in each hemisegment of the embryo. Normally these axons project anteriorly on the ipsilateral side to form a distinct Apterous fascicle (Fig. 3I, arrow) (Lundgren et al. 1995). In embryos of the genotype C155; UAS-AcGq3, axons from Apterous-expressing neurons no longer remain on the ipsilateral side but are now able to cross the midline (Fig. 3J, arrows). However, unlike axons that crossover in robo mutant embryos (Wolf and Chiba, 2000), these appear to stall after reaching and crossing the midline.

\section{Expression of $A c G q 3$ in specific neurons leads to aberrant midline crossing}

The phenotypes observed in embryos expressing AcGq3 suggest that $\mathrm{Gq}$ signaling can drive formation of the commissures and longitudinal tracts. This idea is supported by the phenotype observed in embryos homozygous for $D f(2 R) v g-C$ (which uncovers $d g q$ ) (Fig. 2J). In these embryos the commissures appear thinner, and there are extensive breaks in the longitudinal tracts. These phenotypes are considerably stronger than those observed for frazzled mutants, which is also uncovered by the same deficiency, indicating that the effect of removing both Dgq and Frazzled is additive. However, these defects could be either caused by erroneous signaling within neurons so that they misinterpret existing cues, or by a non-autonomous mechanism that affects midline guidance cues. The latter would result in misplaced neurons or glia or neurons with changed identity. In $D f(2 R) v g-C$ embryos, the pattern of neurons expressing the Even-skipped (Eve) protein appear normal (data not shown), indicating that the defects seen occur after neuronal patterning is complete.

To confirm that the phenotype seen by expression of AcGq3 in the CNS is caused by altered signaling within neurons expressing AcGq3, we used more restrictive GAL4 drivers to express $U A S$ $A c G q 3$ in specific subsets of neurons of the embryonic CNS. $f t z_{\text {ng }}-G A L 4$ expresses in a small subset of neurons that include mostly motor neurons and some interneurons like vMP2, pCC, dMP2, and MP1 (Doe et al., 1988; Landgraf et al., 1999). These interneurons pioneer the longitudinal axon tracts, which stain positive for Fasciclin II. In addition, these axons never cross the midline. On expressing $U A S-A c G q 3$ with $f t z_{n g}-G A L 4$, midline crossing by Fasciclin II-positive axons could be observed. At stage 13, the pCC axon which, normally projects anteriorly on the ipsilateral side, could be seen turning toward the midline (Fig. $4 B$ ). At stage 16 , aberrant midline crossing by the medial fascicle could be observed (data not shown). The number of midline crossovers at this stage is less as compared with C155-GAL4, presumably because of the restricted and comparatively weak expression of the $f t z_{n g}-G A L 4$ line (Table 1). Similar results were obtained with eve $e_{n g}-G A L 4$, which expresses in aCC, pCC, and RP2 neurons (Fig. 4C,D) (Baines et al., 1999; Featherstone et al., 2000). The pCC axon can be seen crossing the midline, whereas the aCC and RP2 projections look normal on expression of AcGq3 (Fig. 4D). Axons from Apterous-expressing dorsal cells (dc) can also change their trajectory on expression of AcGq3 (Fig.

(arrow); $v c$, ventral cell; $d c$, dorsal cell. $J$, Stage 16. Embryo of the genotype C155-GAL4/UAS-AcGq3; Ap-taußgal/+. Axons derived from apterous-expressing lateral cell $(l c)$ and dorsal cell $(d c)$ cross the midline and stall (arrows). T2, Second thoracic segment; T3, third thoracic segment; $A 1$, first abdominal segment. All photographs were taken at a magnification of $200 \times$ 


\begin{tabular}{|c|c|c|c|}
\hline Genotype & Stalling phenotype & Midline crossover & $\%$ Midline crossover \\
\hline$C 155-G A L 4 / U A S-A c G q 3$ & $106 / 308 *(34.41 \%)$ & $64 / 133 * *$ & 48.10 \\
\hline$U A S-A c G q 3 ;+/+; f t z_{n g}-G A L 4$ & $171 / 854 *(20.02 \%)$ & $86 / 427 * *$ & 20.14 \\
\hline$U A S-A c G q 3 ; r^{\prime} o b o^{1} /+; f t z_{n g}-G A L 4$, UAS-taußgal & & $316 / 623 * *(5.35)^{+}$ & 50.7 \\
\hline $\operatorname{robo}^{1} /+$ & & $12 / 245^{* *}(2.10)^{+}$ & 4.8 \\
\hline C155-GAL4/+; UAS-AcGq3/+; UAS-roboY-F/+ & & $0 / 161^{* *}$ & 0.0 \\
\hline C155-GAL4/UAS-AcGq3;fra/CyoActßgal & & $37 / 259 * *$ & 15.3 \\
\hline C155-GAL4/UAS-AcGq3; $\mathrm{fra}^{3} / \mathrm{fra}^{4} ;+/+$ & & $13 / 245^{* *}$ & 5.3 \\
\hline
\end{tabular}

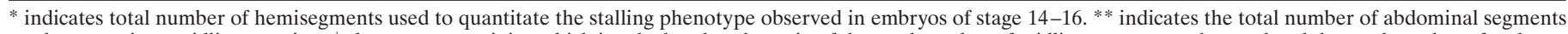

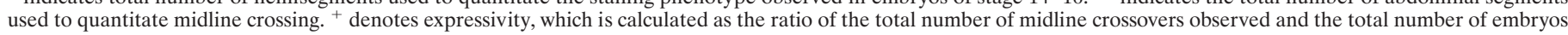
showing at least one crossover.

$4 E, F)$. Instead of projecting toward the anterior and in an ipsilateral direction as is normal (Fig. $4 E, F$, asterisks), a fraction of the axons can be seen drifting across the midline (Fig. $4 F$, arrowhead). The autonomy of AcGq3 function is further supported by the observation that neurons and glia are patterned normally in $C 155-G A L 4 / U A S-A c G q 3$ embryos, as judged by staining with anti-Eve and anti-Repo antibodies (data not shown). Taken together these data demonstrate that specific activation of Dgq $\alpha 3$ in ipsilaterally projecting neurons causes changes in their axonal trajectories so that they are now able to project across the midline.

\section{Midline crossing by ectopic expression of AcGq3 is independent of Robo downregulation}

To understand how Dgq $\alpha 3$ acts to change axonal paths, we looked for possible interactions with genes known to affect midline guidance. Axons that cross the midline and project along the contralateral longitudinal tract normally need to downregulate expression of Robo, which acts as a receptor for the midline repellant Slit (Kidd et al., 1999). It is known that Robo downregulation requires Commissureless, but the precise mechanism is not understood (Tear et al., 1996; Kidd et al., 1998b). A possible mechanism by which AcGq3 could promote midline crossing was by downregulating Robo. To test this hypothesis we looked at Robo expression in $f t z_{n g}-G A L 4 ; U A S-A c G q 3$ embryos. Interestingly, we find that Robo is not downregulated visibly in axons that ectopically cross the midline under the influence of AcGq3 (Fig. 5B). The extent of Robo staining seen on these axons that aberrantly cross the midline is comparable with that seen on the longitudinal tracts. Thus, constitutive activation of Dgq $\alpha 3$ results in aberrant midline crossing of axons by a mechanism that is independent of Robo downregulation.

\section{Reducing robo function enhances midline crossing by AcGq3}

Another mechanism by which AcGq3 could induce midline crossing is through inhibition of the repulsive signal mediated by Robo. If this were so, then reducing levels of Robo by genetic means should enhance the phenotype of AcGq3. To test this, AcGq 3 was expressed using $f t z_{n g}-G A L 4$ in embryos carrying a single copy of the robo ${ }^{1}$ mutant allele. robo ${ }^{1}$ is a recessive mutation. However, embryos with one copy of this mutation show midline crossing at a frequency of $\sim 10 \%$ (Fritz and VanBerkum, 2000) (Fig. 5C, Table 1). When $U A S$ - $A c G q 3 ;$ robo $^{1} /+;$ ftz $_{n g}-G A L 4$ embryos were stained with mAb 1D4, a significant increase in the number of midline crossovers was observed as compared with embryos of the genotype $U A S-A c G q 3 ;+/+; f t z_{n g}-G A L 4$ (Fig. $5 D$, Table 1) This suggests that activation of Dgq $\alpha 3$ antagonizes the repulsive output through Robo resulting in excessive midline crossing. The antagonism could be mediated either through phosphorylation of Robo or signaling components that function downstream and/or in parallel with Robo.

Phosphorylation of a single tyrosine residue on Robo by Abelson (Abl) tyrosine kinase inhibits Robo repulsive signaling and is needed for normal midline crossing to take place. Expression of a mutant form of Robo in which this tyrosine residue (Y1040) has been replaced with a phenylalanine (in a transgenic strain referred to as $U A S$-roboY-F), lead to constitutive Robo signaling such that no axons cross the midline, resulting in a complete absence of commissure formation (Bashaw et al., 2000). If AcGq3 acts upstream of Robo, we predicted that ectopic midlinecrossovers, induced by expression of $\mathrm{AcGq} 3$, would be reduced in presence of Robo Y-F. In fact, in embryos expressing both AcGq3 and Robo Y-F, no ectopic crossovers are seen (Fig. 5F, Table 1), indicating that AcGq3 could inhibit Robo signaling by promoting Robo phosphorylation. This finding is also supportive of the fact that $\mathrm{AcGq} 3$ exerts its effect independent of Commissureless-mediated Robo downregulation. It is possible however, that AcGq3 acts through a parallel pathway that is no longer effective in the presence of Robo Y-F (see Discussion).

\section{Ectopic midline crossing requires Frazzled function}

Both the spatiotemporal pattern of expression and functional analysis of $d g q$ indicate that $\mathrm{Gq}$ activation in vivo promotes midline crossing. Axons that cross the midline need to turn down their repulsive signaling pathway(s) as well as respond positively to attractive cues. We therefore looked to see if changes in the levels of "attractive" signaling such as the Netrin-Frazzled pathway affect the phenotype of AcGq3. Interestingly, AcGq3 phenotype shows a dosage-dependent interaction with Fra. Removal of a single copy of the Fra gene led to a threefold reduction in the number of midline crossovers induced by AcGq3 (Table 1). A further reduction was observed on removal of both copies of the Fra gene as seen in embryos of the genotype C155-GAL4/UAS$A c G q 3 ; \mathrm{fra}^{3} / \mathrm{fra}^{4}$ (Table 1, Fig. 6C). Signaling through AcGq3 is thus sensitive to levels of Frazzled in the CNS.

To examine the effect, if any, of AcGq3 on the frazzled mutant phenotype, embryos of the genotype C155-GAL4/UAS-AcGq3; $\mathrm{fra}^{3} / \mathrm{fra}^{4}$ were examined with anti-connectin antibody (Fig. 6) and BP102 (data not shown). Anti-connectin labels a distinct axon fascicle in the longitudinal connectives, axon projections of SP1 and RP1 neurons that project through the anterior commissure, 


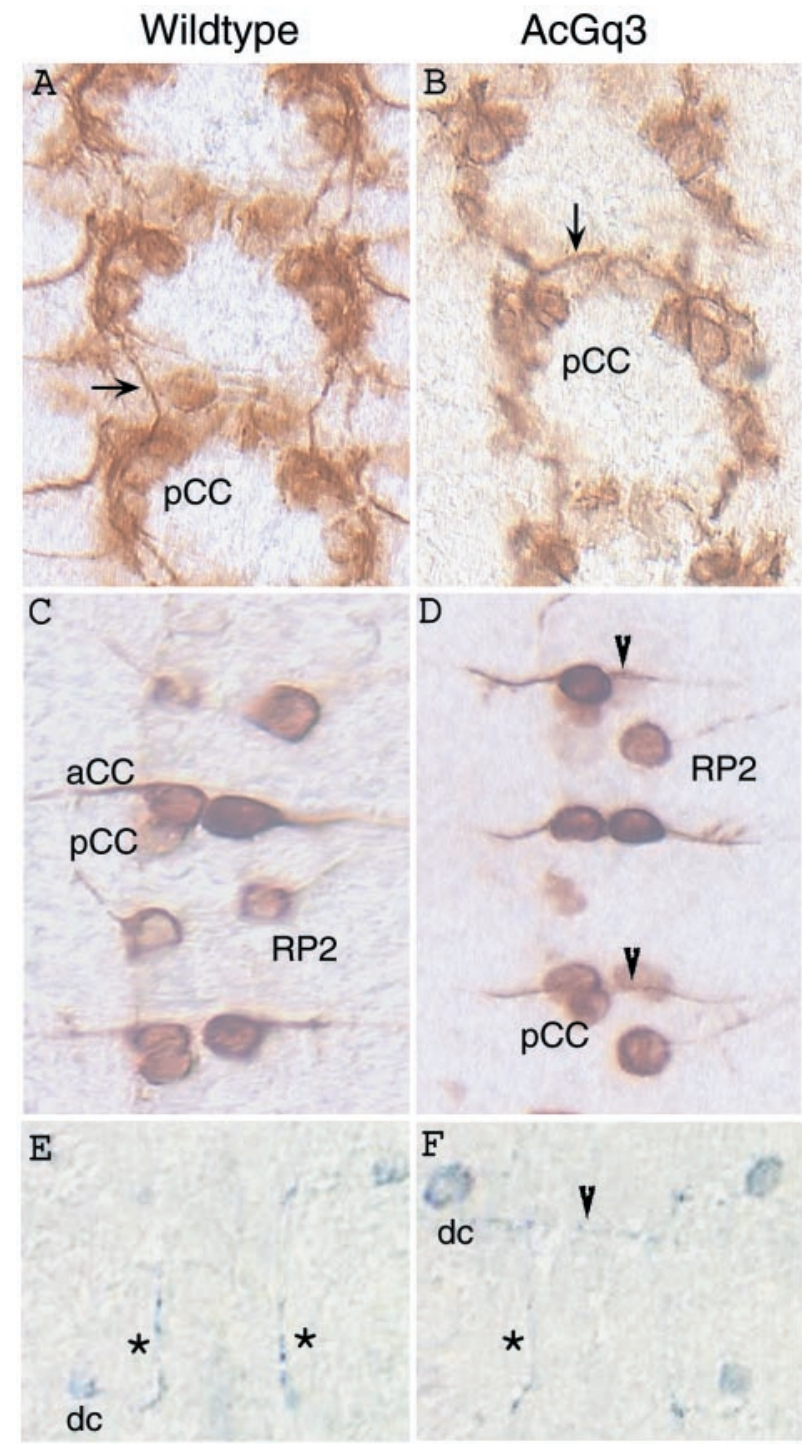

Figure 4. Midline crossing by ectopic expression of AcGq3 appears cell autonomous. $A, B$, Embryos stained with anti-fasciclin II. $C-F$, Embryos stained with anti- $\beta$ gal antibody. $A, f t z_{n g}-G A L 4 /+$ control embryo. $B$, Embryo of the genotype UAS-AcGq3/+; ftz ${ }_{n g}-G A L 4 /+$. A, Late stage 13 embryo shows ipsilateral projection of the pCC axon (arrow). B, Early stage 13 embryo. Arrow indicates projection of the pCC axon toward the midline. $C$, UAS-taußgal;eve-GAL4 embryo at stage 16 embryo. The pCC axon is not visible in this photomicrograph because it runs at a different focal plane from the cell bodies. $D$, UAS-AcGq3; UAS-taußgal;eve-GAL4 embryo at stage 16 . The pCC axon is seen projecting across the midline (arrowheads). Because eve-GAL4 expression was not consistent in each segment, quantification of this phenotype (Table 1) was done on the basis of UAS-taußgal expression. E, F, Abdominal segments of stage $15 \mathrm{em}-$ bryos of the genotype $A p-G A L 4 \mid A c G q 3^{F 58 c}$; apC-taußgal/+. Normal longitudinal fascicles, projecting from the dorsal cell $(d c)$, are marked with an asterisk, whereas the arrowhead in $F$ shows an axon crossing the midline.

and a subset of axons that project through the posterior commissure to their contralateral targets (Fig. 6D, arrowhead) (Meadows et al., 1994). In embryos of the genotype C155-GAL4/+; $\mathrm{fra}^{3} / \mathrm{fra}^{4}$, breaks were observed in connectin-positive commissural axons and longitudinal tracts (Fig. 6E, arrowhead). Embryos of the genotype C155-GAL4/UAS-AcGq3; $\mathrm{fra}^{3} / \mathrm{fra}^{4}$ also show similar breaks (Fig. $6 F$, arrowhead), indicating that AcGq3 does not have an effect on the frazzled mutant phenotype. Similar results were obtained by staining with BP102.
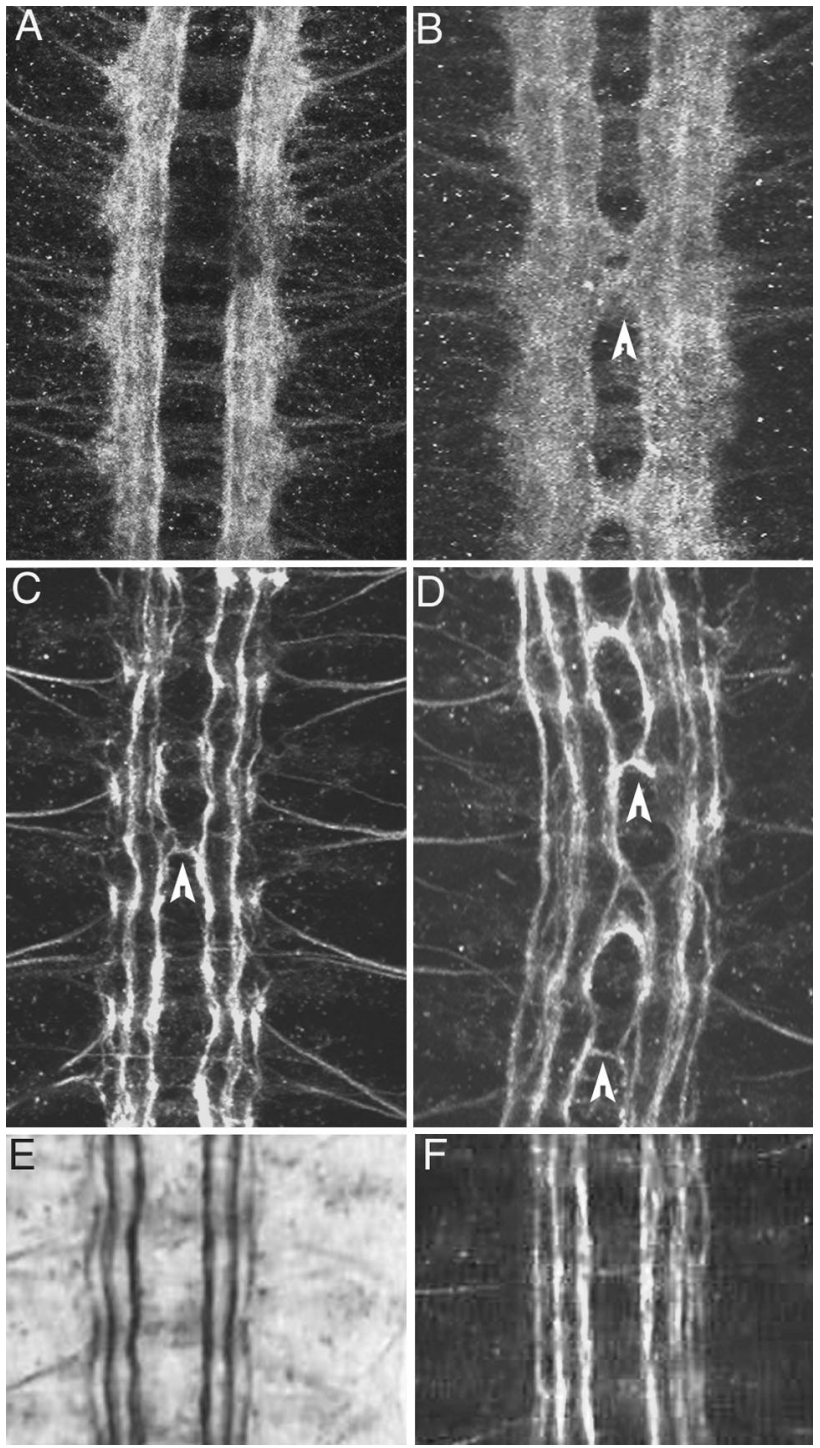

Figure 5. AcGq3 inhibits repulsive signaling by Robo through a mechanism independent of Robo downregulation. $A-D$ are confocal images of stage 16 embryos stained with antibodies against Robo $(A, B)$ and Fasciclin II $(C, D) . E$ and $F$ are immunohistochemical and fluorescent images, respectively, of stage 16 embryos stained with antibodies against Fasciclin II. $A$, In a control embryo of the genotype $f t z_{n g}-G A L 4 /+$, Robo expression is confined to axons in the longitudinal tracts. $B$, An embryo of the genotype UAS-AcGq3/+;ftz ${ }_{n g}-G A L 4 /+$. Robo expression is observed in ectopic commissural axons (arrowhead). $C$, An embryo of the genotype $U A S-A c G q 3 /+; f t z_{n g}-G A L 4 /+$. Arrowhead points to a single midline crossover. D, UAS-AcGq3/+;robo ${ }^{1} /+; f t z_{n g} G A L 4 /+$ embryo with enhanced midline crossovers (arrowheads). E, A stage 16 embryo of the genotype $C 155-G A L 4 /+$; UAS-roboY-F. F, A stage 16 embryo of the genotype C155-GAL4/+, AcGq3/+; UAS-roboY-F/+.

\section{DISCUSSION}

\section{Embryonic expression of Dgq $\alpha 3$}

$D g q$ was originally identified from a head cDNA library as a homolog of mammalian G $\alpha$ q (Strathmann and Simon, 1990). Initial functional characterization suggested that it was a visualspecific G-protein essential for Drosophila visual transduction (Lee et al., 1990, 1994; Scott et al., 1995). However, from subsequent studies it was apparent that splice variants of $d g q$ existed in other adult tissues (Talluri et al., 1995; Alvarez et al., 1996). In this study we have analyzed $d g q$ expression and function during 

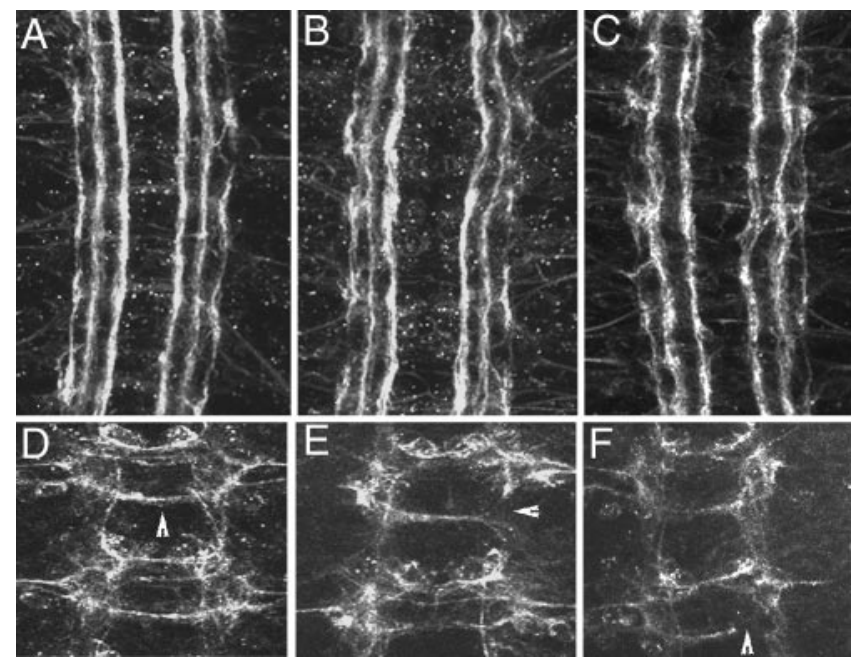

Figure 6. Frazzled function is essential for ectopic midline crossing induced by $U A S-A c G q 3$. $A-C$, Stage 16 embryos stained with mAb 1D4. $D-F$, Stage 14 embryos stained with antibodies against Connectin. $A$, In wild-type embryos, three distinct fascicles can be observed on either side of the midline. B, An embryo of the genotype $C 155-G A L 4 /+; \mathrm{ra}^{3} / \mathrm{fra}^{4}$. C, An embryo of the genotype C155-GAL4/UAS-AcGq3; $\mathrm{fra}^{3} / \mathrm{fra}^{4} ;+/+$. Aberrant midline crossing by ipsilateral axons is absent. $D, C 155-G A L 4 /+$; $\mathrm{fra} /$ CyoAct $\beta g a l$ embryo at stage 14 showing the wild-type staining pattern of anti-Connectin antibody (Meadows et al., 1994). A Connectin-positive axon is seen projecting through the posterior commissure (arrowhead). E, Embryo of the genotype C155-GAL4/+; $\mathrm{ra}^{3} / \mathrm{fra}^{4} ; \mathrm{F}, \mathrm{C155-GAL4/UAS-}$ $A c G q 3 ; \mathrm{fra}^{3} / \mathrm{fra}^{4} ;+/+$ embryo, show breaks in connectin positive commissural axon (arrowheads).

development of the Drosophila embryonic CNS. From analysis of $d g q$ transcripts and protein we have shown that the $d g q \alpha 3$ splice variant is the primary embryonic form, suggesting multifunctional roles for this protein. Considering the broad expression pattern of $d g q$, a traditional mutagenesis approach might be unable to address late developmental phenotypes caused by $d g q \alpha 3$ loss-of-function. The UAS-GAL4 system offered an alternate strategy that allowed us to dissect $d g q \alpha 3$ function during axon guidance. $U A S$ - $A c G q 3$ essentially functions as a dominant gain-of-function allele in a tissue- and cell-specific manner.

\section{Function of Dgq $\alpha 3$ in the embryonic CNS}

The induction of ectopic midline crossing by AcGq3 suggests that Dgq $\alpha 3$ function might be required during commissural growth. What activates $\operatorname{Dgq} \alpha 3$ in vivo? In Drosophila, the only pathway so far known to mediate attraction toward the midline, is the NetrinFrazzled signaling pathway. However, null mutants for netrins and frazzled continue to show formation of commissures, albeit thin and poorly organized. The failure to show a complete absence of commissures suggests that an alternate signaling pathway or pathways exists at the midline that promotes commissural growth. The presence of a second attractive signaling pathway operating at the midline has also been suggested based on analysis of mutants involved in formation of commissures (Hummel et al., 1999a,b). Dgq $\alpha 3$ might act as a component of this alternate pathway to promote commissural growth.

Signaling mechanisms involved in DCC/Frazzled-mediated attraction are poorly understood in vertebrates as well as invertebrates. In vitro studies using pharmacology in vertebrate systems have shown that guidance mediated by Netrin-1 is dependent on cAMP levels in the growth cone. Increase in cAMP levels results in attraction, whereas low levels of the cyclic nucleotide causes repulsion (Song et al., 1997). In Xenopus cultured neurons, Netrin-1-induced turning response has also been shown to depend on $\mathrm{Ca}^{2+}$ influx through the plasma membrane and $\mathrm{Ca}^{2+}$ induced $\mathrm{Ca}^{2+}$ release through intracellular stores (Hong et al., 2000). The involvement of second messengers such as $\mathrm{Ca}^{2+}$ and cAMP suggests that G-protein-coupled signaling pathways might be involved. Heterotrimeric G-proteins are also thought to play a role in neuronal migration (Horgan et al., 1994) and growth cone collapse (Nakayama et al., 1999). A study implicating the Adenosine A2b receptor in Netrin-1 signaling supports this idea (Corset et al., 2000). More recently however, it has been shown that DCC can bind Netrin-1 and signal attraction independent of the Adenosine A2b receptor (Stein et al., 2001). This study shows that DCC undergoes a ligand-dependent dimerization essential for its signaling that remains unaffected even in the presence of antagonists to adenosine receptors, thus providing evidence that DCC alone is central to Netrin-1 signaling. As compared with vertebrates, the mechanism of Netrin signaling in Drosophila is still obscure. Recent studies involving this signaling pathway have, however, suggested that a second receptor for Netrins could exist in the nervous system (Gong et al., 1999; Hiramoto et al., 2000). Given the evolutionarily conserved nature of both, the ligand and the receptor, similar downstream signaling elements are very likely involved in mediating attraction. It is possible that a seven transmembrane domain receptor activates Dgq $\alpha 3$ signaling in response to novel attractive cues or Netrins leading to increase in $\mathrm{Ca}^{2+}$ levels and thus promoting attraction.

Our results from the genetic analysis of $A c G q 3$ and frazzled suggest that Frazzled function is essential for AcGq3-mediated ectopic midline crossing. In addition, they also indicate that $\operatorname{Dgq} \alpha 3$ does not function downstream of frazzled signaling. A simple explanation for these observations could be that activity of Dgq $\alpha 3$ and Frazzled are both essential to promote midline crossing. The effects of the two signaling pathways are additive; activation of Frazzled and Dgq $\alpha 3$ are both necessary to elicit attraction. Removal of one or both copies of frazzled in the presence of AcGq3 simply reduces the sum total of attraction sensed by the growth cone, thus inhibiting aberrant midline crossing of ipsilateral axons.

\section{Interaction of $\mathrm{AcGq3}$ with robo}

The antagonism between AcGq3 and Robo suggests that AcGq3 operates by modulating repulsion from the midline during commissural growth. It has been demonstrated that Robo signaling is negatively modulated by tyrosine phosphorylation by Abelson kinase (Bashaw et al., 2000). Our results in Figure 5 suggest that AcGq3 could inhibit Robo signaling by a similar mechanism of phosphorylating Robo. It could perhaps do this by activating a kinase cascade involving a nonreceptor tyrosine kinase such as Bruton's tyrosine kinase (BTK or Tec kinase) which, in mammalian cells, has been shown to be a direct effector of Gq signaling (Bence et al., 1997; Ma and Huang, 1998). Our results are equally consistent with the possibility that AcGq3 and Robo act through parallel pathways, such that AcGq3 induced midline crossing requires downregulation of Robo signaling.

Based on the results obtained from genetic analysis of $A c G q 3$ with frazzled and robo, the following models can be proposed to explain the function of $\operatorname{Dgq} \alpha 3$. In the first, $\operatorname{Dgq} \alpha 3$ can be thought of as being a component of the attractive signaling pathway alone. Expression of the activated form of the protein functions to override the repulsive cues at the midline and promote ectopic midline crossing. In such a scenario, one would argue that the 
synergism observed between $A c G q 3$ and robo $^{1}$ is a consequence of the combined effect of reduced Robo signaling and excess attractive signaling induced by $\mathrm{AcGq} 3$ leading to an increase in the number of midline crossovers. In the presence of $U A S$ RoboY-F, repulsive signaling increases to a level that cannot be overriden by AcGq3-attractive signaling. A second possibility is that $\operatorname{Dgq} \alpha 3$ is a component of an attractive signaling pathway, which functions to potentiate Frazzled signaling by negatively modulating the repulsion mediated by Robo signaling. This could be through phosphorylation of Robo. A recent study using spinal axons from stage 22 Xenopus embryos has shown that the repulsive ligand Slit can "silence" the Netrin-mediated attraction through a direct physical interaction between the cytoplasmic domains of Robo and Frazzled (Stein and Tessier-Lavigne, 2001). This ligand-dependent silencing effect serves to promote repulsion of growth cones from the midline during the development of commissures. Dgq $\alpha 3$ might function conversely at the level of downstream effector molecules to inhibit repulsion in response to attractive cues to promote midline crossing.

In summary, our results predict the involvement of a Gqmediated signaling pathway in regulating midline crossing in Drosophila. In addition, they also support the notion that balance between attraction and repulsion is a crucial factor that determines the final response of a growth cone to different cues. Inhibition of $d g q$ function specifically in the growth cones should prove useful in dissecting out other components of this pathway that regulates midline crossing.

\section{REFERENCES}

Alvarez CE, Robison K, Gilbert W (1996) Novel Gq alpha isoform is a candidate transducer of rhodopsin signaling in a Drosophila testesautonomous pacemaker. Proc Natl Acad Sci USA 93:12278-12282.

Baines RA, Robinson SG, Fujioka M, Jaynes JB, Bate M (1999) Postsynaptic expression of tetanus toxin light chain blocks synaptogenesis in Drosophila. Curr Biol 9:1267-1270.

Bashaw GJ, Goodman CS (1999) Chimeric axon guidance receptors: the cytoplasmic domains of slit and netrin receptors specify attraction versus repulsion. Cell 97:917-926.

Bashaw GJ, Kidd T, Murray D, Pawson T, Goodman CS (2000) Repulsive axon guidance: Abelson and Enabled play opposing roles downstream of the roundabout receptor. Cell 101:703-715.

Bence K, Ma W, Kozasa T, Huang XY (1997) Direct stimulation of Bruton's tyrosine kinase by G(q)-protein alpha-subunit. Nature 389:296-299.

Bonkowsky JL, Yoshikawa S, O'Keefe DD, Scully AL, Thomas JB (1999) Axon routing across the midline controlled by the Drosophila Derailed receptor. Nature 402:540-544.

Brand AH, Perrimon N (1993) Targeted gene expression as a means of altering cell fates and generating dominant phenotypes. Development 118:401-415.

Chan SS, Zheng H, Su MW, Wilk R, Killeen MT, Hedgecock EM, Culotti JG (1996) UNC-40, a C. elegans homolog of DCC (Deleted in Colorectal Cancer), is required in motile cells responding to UNC-6 netrin cues. Cell 87:187-195.

Corset V, Nguyen-Ba-Charvet KT, Forcet C, Moyse E, Chedotal A, Mehlen P (2000) Netrin-1-mediated axon outgrowth and cAMP production requires interaction with adenosine $\mathrm{A} 2 \mathrm{~b}$ receptor. Nature 407:747-750.

Culotti JG, Merz DC (1998) DCC and Netrins. Curr Opin Cell Biol 10:609-613.

DeVivo M, Chen J, Codina J, Iyengar R (1992) Enhanced phospholipase C stimulation and transformation in NIH-3T3 cells expressing Q209L Gq $\alpha$ subunits. J Biol Chem 267:18263-18266.

Doe CQ, Hiromi Y, Gehring WJ, Goodman CS (1988) Expression and function of the segmentation gene fushi tarazu during Drosophila neurogenesis. Science 239:170-175.

Edery I, Zwiebel LJ, Dembinska ME, Rosbash M (1994) Temporal phosphorylation of the Drosophila period protein. Proc Natl Acad Sci USA 91:2260-2264.

Exton JH (1994) Phosphoinositide phospholipases and G proteins in hormone action. Annu Rev Physiol 56:349-369.

Featherstone DE, Rushton EM, Hilderbrand-Chae M, Phillips AM, Jackson FR, Broadie K (2000) Presynaptic glutamic acid decarboxylase is required for induction of the postsynaptic receptor field at a glutamatergic synapse. Neuron 27:71-84.

Fritz JL, VanBerkum MF (2000) Calmodulin and son of sevenless dependent signaling pathways regulate midline crossing of axons in the. Drosophila CNS Development 127:1991-2000.

Gong Q, Rangarajan R, Seeger M, Gaul U (1999) The netrin receptor frazzled is required in the target for establishment of retinal projections in the Drosophila visual system. Development 126:1451-1456.

Goodman CS, Doe CQ (1993) Embryonic development of the Drosophila CNS. In: The development of Drosophila melanogaster (Bate M, Martinez-Arias A, ed), pp 1131-1206. Cold Spring Harbor, NY: Cold Spring Harbor Laboratory.

Gould AP, Lai RYK, Green MJ, White RAH (1990) Blocking cell division does not remove the requirement for polycomb function in Drosophila embryogenesis. Development 110:1319-1325.

Harris R, Sabatelli LM, Seeger MA (1996) Guidance cues at the Drosophila CNS midline: identification and characterization of two Drosophila Netrin/UNC-6 homologs. Neuron 17:217-228.

Hasan G, Rosbash M (1992) Drosophila homologs of two mammalian intracellular $\mathrm{Ca}(2+)$-release channels: identification and expression patterns of the inositol 1,4,5-triphosphate and the ryanodine receptor genes. Development 116:967-975.

Hiramoto M, Hiromi Y, Giniger E, Hotta Y (2000) The Drosophila Netrin receptor Frazzled guides axons by controlling Netrin distribution. Nature 406:886-889.

Hong K, Hinck L, Nishiyama M, Poo MM, Tessier-Lavigne M, Stein E (1999) A ligand-gated association between cytoplasmic domains of UNC5 and DCC family receptors converts netrin-induced growth cone attraction to repulsion. Cell 97:927-941.

Hong K, Nishiyama M, Henley J, Tessier-Lavigne M, Poo M (2000) Calcium signalling in the guidance of nerve growth by netrin-1. Nature 403:93-98.

Horgan AM, Lagrange MT, Copenhaver PF (1994) Developmental expression of $G$ proteins in a migratory population of embryonic neurons. Development 120:729-742.

Hummel T, Schimmelpfeng K, Klambt C (1999a) Commissure formation in the embryonic CNS of Drosophila. Dev Biol 209:381-398.

Hummel T, Schimmelpfeng K, Klambt C (1999b) Commissure formation in the embryonic CNS of Drosophila. Development 126:771-779.

Ishii N, Wadsworth WG, Stern BD, Culotti JG, Hedgecock EM (1992) UNC-6, a laminin-related protein, guides cell and pioneer axon migrations in C. elegans. Neuron 9:873-881.

Keino-Masu K, Masu M, Hinck L, Leonardo ED, Chan SS, Culotti JG, Tessier-Lavigne M (1996) Deleted in Colorectal Cancer (DCC) encodes a netrin receptor. Cell 87:175-185.

Kennedy TE, Serafini T, De La Torre JR, Tessier-Lavigne M (1994) Netrins are diffusible chemotropic factors for commissural axons in the embryonic spinal cord. Cell 78:425-435.

Kidd T, Brose K, Mitchell KJ, Fetter RD, Tessier-Lavigne M, Goodman CS, Tear G (1998a) Roundabout controls axon crossing of the CNS midline and defines a novel subfamily of evolutionarily conserved guidance receptors. Cell 92:205-215.

Kidd T, Russell C, Goodman CS, Tear G (1998b) Dosage-sensitive and complementary functions of roundabout and commissureless control axon crossing of the CNS midline. Neuron 20:25-33.

Kidd T, Bland KS, Goodman CS (1999) Slit is the midline repellent for the robo receptor in. Drosophila Cell96:785-794.

Klambt C, Roger Jacobs J, Goodman CS (1991) The midline of the Drosophila central nervous system: a model for the genetic analysis of cell fate, cell migration, and growth cone guidance. Cell 84:801-815.

Kolodziej PA, Timpe LC, Mitchell KJ, Fried SR, Goodman CS, Jan LY, Jan YN (1996) Frazzled encodes a Drosophila member of the DCC immunoglobulin subfamily and is required for CNS and motor axon guidance. Cell 87:197-204.

Landgraf M, Roy S, Prokop A, VijayRaghavan K, Bate M (1999) evenskipped determines the dorsal growth of motor axons in Drosophila. Neuron 22:43-52.

Lee YJ, Dobbs MB, Verardi ML, Hyde DR (1990) dgq: a Drosophila gene encoding a visual system-specific $G$ alpha molecule. Neuron 5:889-898.

Lee YJ, Shah S, Suzuki E, Zars T, O’Day PM, Hyde DR (1994) The Drosophila $d g q$ gene encodes a $\mathrm{G}$ alpha protein that mediates phototransduction. Neuron 13:1143-1157.

Lin DM, Goodman CS (1994) Ectopic and increased expression of Fasciclin II alters motoneuron growth cone guidance. Neuron 13:10551069.

Lundgren SE, Callahan CA, Thor S, Thomas JB (1995) Control of neuronal pathway selection by the Drosophila LIM homeodomain gene apterous. Development 121:1769-1773.

Ma YC, Huang XY (1998) Identification of the binding site for Gqalpha on its effector Bruton's tyrosine kinase. Proc Natl Acad Sci USA 95:12197-12201.

Meadows LA, Gell D, Broadie K, Gould AP, White RA (1994) The cell adhesion molecule, connectin, and the development of the Drosophila neuromuscular system. J Cell Sci 107:321-328. 
Menon KP, Zinn K (1998) Tyrosine kinase inhibition produces specific alterations in axon guidance in the grasshopper embryo. Development 125:4121-4131.

Mitchell KJ, Doyle JL, Serafini T, Kennedy TE, Tessier-Lavigne M, Goodman CS, Dickson BJ (1996) Genetic analysis of Netrin genes in Drosophila: netrins guide CNS commissural axons and peripheral motor axons. Neuron 17:203-215.

Nakayama T, Goshima Y, Misu Y, Kato T (1999) Role of cdk5 and tau phosphorylation in heterotrimeric $\mathrm{G}$ protein-mediated retinal growth cone collapse. J Neurobiol 41:326-339.

Rajagopalan S, Vivancos V, Nicolas E, Dickson BJ (2000a) Selecting a longitudinal pathway. Robo receptors specify the lateral position of axons in the Drosophila CNS. Cell 103:1033-1045.

Rajagopalan S, Nicolas E, Vivancos V, Berger J, Dickson BJ (2000b) Crossing the midline: roles and regulation of Robo receptors. Neuron 28:767-777.

Sambrook J, Fritsch EF, Maniatis T (1989) Molecular cloning: a laboratory manual. Cold Spring Harbor, NY: Cold Spring Harbor Laboratory.

Sanger F, Nicklen S, Coulson AR (1977) DNA sequencing with chainterminating inhibitors. Proc Natl Acad Sci USA 74:5463-5467.

Schmucker D, Taubert H, Jackle H (1992) Formation of the Drosophila larval photoreceptor organ and its neuronal differentiation require continuous Kruppel gene activity. Neuron 6:1025-1039.

Scott K, Becker A, Sun Y, Hardy R, Zuker CS (1995) Gq alpha protein function in vivo: genetic dissection of its role in photoreceptor cell physiology. Neuron 15:919-927.

Simpson JH, Kidd T, Bland KS, Goodman CS (2000a) Short-range and long-range guidance by slit and its Robo receptors. Robo and Robo2 play distinct roles in midline guidance. Neuron 28:753-766.

Simpson JH, Bland KS, Fetter RD, Goodman CS (2000b) Short- range and long-range guidance by Slit and its Robo receptors. A combinatorial code of Robo receptors controls lateral position. Cell 103:10191032.

Sinha M, Hasan G (1999) Sequencing and exon mapping of the inositol 1,4,5-trisphosphate receptor cDNA from Drosophila embryos suggests the presence of differentially regulated forms of RNA and protein. Gene 233:271-276.
Song HJ, Ming GL, Poo MM (1997) cAMP-induced switching in turning direction of nerve growth cones. Nature 388:275-279.

Stein E, Tessier-Lavigne M (2001) Hierarchical organization of guidance receptors: silencing of netrin attraction by slit through a Robo/ DCC receptor complex. Science 291:1928-1938.

Stein E, Zou Y, Poo M, Tessier-Lavigne M (2001) Binding of DCC by netrin-1 to mediate axon guidance independent of adenosine A2B receptor activation. Science 291:1976-1982.

Strathmann M, Simon MI (1990) G protein diversity: a distinct class of alpha subunits is present in vertebrates and invertebrates. Proc Natl Acad Sci USA 87:9113-9117.

Sun Q, Bahri S, Schmid A, Chia W, Zinn K (2000) Receptor tyrosine phosphatases regulate axon guidance across the midline of the Drosophila embryo. Development 127:801-812.

Talluri S, Bhatt A, Smith DP (1995) Identification of a Drosophila G protein alpha subunit (dGq alpha-3) expressed in chemosensory cells and central neurons. Proc Natl Acad Sci USA 92:11475-11479.

Tautz D, Pfiefle C (1989) A non radioactive in situ hybridization method for localisation of RNAs in Drosophila embryos reveals translation control of the segmentation gene hunchback. Chromosoma 98:81-85.

Tear G, Harris R, Sutaria S, Kilomanski K, Goodman CS, Seeger MA (1996) Commissureless controls growth cone guidance across the CNS midline in Drosophila and encodes a novel membrane protein. Neuron 16:501-514.

Tessier-Lavigne M, Goodman CS (1996) The molecular biology of axon guidance. Science 274:1123-1133.

Wang Q, Hasan G, Pikielny CW (1999) Preferential expression of biotransformation enzymes in the olfactory organs of Drosophila melanogaster, the antennae. J Biol Chem 274:10309-10315.

Wieschaus F, Nusslein-Volhard C (1986) Looking at embryos. In: Drosophila: a practical approach (Roberts DB, ed), pp 199-227. Oxford: IRL.

Wolf BD, Chiba A (2000) Axon pathfinding proceeds normally despite disrupted growth cone decisions at CNS midline. Development 127:2001-2009.

Zheng JQ (2000) Turning of nerve growth cones induced by localized increases in intracellular calcium ions. Nature 403:89-93. 